2. G. J. Withrow, The Natural Philosophy of Time. XII + 324 p. Thomas Nelson \& Sons Ltd., London/Edinburg 1961.

Nachdem durch die Probleme der «Zeitumkehr» und der möglichen Asymmetrie der Zeit die «Naturgeschichte» der Zeit oder das Zeitproblem in der modernsten Physik wieder besonders aktuell geworden sind, wird man vorliegende kenntnisreiche und tiefgründende Darstellung sehr begrüßen. Sie hat seit ihrem Erscheinungsdatum * nichts an Bedeutung eingebüßt, was schon ihren hohen Wert erweist. Zudem stellt sie in mancher Hinsicht eine reife Lebensleistung dar.

Sie erwuchs aus der vielseitigen philosophischen, physikalischen, technischen und psychologischen Untersuchung des Zeitbegriffes und aus der Überzeugung, daß die Zeit nichts Vorgegebenes, sondern etwas zu Erforschendes sei, unter Zugrundelegung der geschichtlichen Entwicklung der «Theorie» und der Erfahrung der Zeit.

Grundsätzlich ist die Zeitbestimmung entweder ein fundamentaler Sachverhalt oder sie wird nach Möglichkeit «eliminiert». Im Mittelpunkt steht im ersten Fall das «Werden»: eine Vorstellung, für welche die Idee des Raumes kein Analagon bietet. Die Zeit ist daher stets dynamisch (wahrscheinlich ist es der Raum auch; doch hat man diesen Aspekt selten ernst genommen).

Die gegenwärtige Konzeption der Zeit ist überaus komplex; sie ist es, wie diese Untersuchung zeigt, noch weit mehr als auch das beste «Zeitbewußtsein» den Tatbestand realisiert; vor allem die «biologische Zeit», aber auch die "physikalische Zeit» mit ihren stets kürzeren Intervallen in der Elementarpartikeloder Quarksphysik (bis zu einer «Zeitdauer» von $10^{-20} \mathrm{~s}$ oder noch weniger) sollten zum Nachdenken führen. Die «verflossene Zeit» in Physik und Biologie steht oft in einem sonderbaren «Verhältnis» zu historischen, also menschlichen Abläufen in der Vergangenheit zugeordneten, Perioden (übrigens ist das Thema «Zeit und Zeitalter in der Geschichte» eine der wenigen hier kaum berührten Aspekte).

Dagegen ist das Problem des «nun und jetzt», bzw. der «Gleichzeitigkeit», auch in der Einsteinschen Relativitätstheorie, sachgerecht erörtert, ebenso die «Kosmische Zeit» unter Annahme des sich ständig ausdehnenden Universums. Mit Recht wird darauf hingewiesen, ob es angeht, die - auch bei Einstein noch anzutreffende - Vorstellung der «Lokalzeit» mit der Vorstellung der Zeitidentität im expandierenden Weltall zu verwenden (S. 237/38), nachdem bereits de Sitter, Eddington, Hubble u. a. auf die Inkonsequenz hinwiesen, und bei Withrow richtig eingeordnet werden. Faszinierend wirkt ferner der vorangehende große Abschnitt über die «mathematische Zeit». Nur recht wenige Gelehrte wären imstande gewesen, ihn so klarsichtig und mit so umfassender historischer Quellenkenntnis zu schreiben; man denkt manchmal an die Leistungen von Hermann Weyl.

\title{
Eduard Fueter
}

* Sie kam dem Rezensenten erst vor Jahresfrist zur Besprechung zu. 